\title{
Hypertrophic Cardiomyopathy and Sports
}

\author{
Ivan Maznev, PhD \\ Antoaneta Dimitrova, PhD \\ Department of Physiotherapy and Rehabilitation, National Sports Academy \\ "Vassil Levski", Sofia, Bulgaria
}

Doi: 10.19044/esj.2018.v14n9p1 URL:http://dx.doi.org/10.19044/esj.2018.v14n9p1

\begin{abstract}
Hypertrophic cardiomyopathy (HCM) is one of the most common causes of sudden cardiac death (SCD) in athletes. Knowledge of the problem is essential for detection of the disease. According to the current recommendations of the European Society of Cardiology (ESC), in individuals with established HCM disease, including those without phenotypic expression, participation in competitive sports is forbidden. The recommendations of the American College of Cardiology/American Heart Association (ACC/AHA) are more liberal, allowing participation in competitive sports in people with non-phenotypic manifestations of the disease and participation in low-intensity sports for patients with phenotypic manifestation. An individual approach will most likely be adopted in the future, mainly on the basis of precise risk stratification.
\end{abstract}

Keywords: Athletes, hypertrophic cardiomyopathy, sudden cardiac death

\section{Introduction}

For the first time in 1958 the English pathologist Donald Teare described the disease as asymmetric left ventricular hypertrophy (LVH) in eight adolescents (Teare, 1958). Later, the disease was called hypertrophic cardiomyopathy. According to the ESC, HCM is defined by the presence of increased left ventricular wall thickness that is not solely explained by increased ventricular wall tension (Elliott et al., 2014). According to the ACA/AHA definition, this is a disease characterized by an unexplained LVH, without the presence of dilation, in the absence of any other cardiac or systemic disease, which in itself could lead to hypertrophy (Gersh et al., 2011). According to the second definition, only genetically determined forms of the disease are taken into account.

A number of studies conducted in different regions of the world show the frequency of $\mathrm{HCM}$ as around $0.2 \%$ of the total population, with no 
significant differences between the different ethnic groups and gender dependence (Maron, 2004). This percentage does not take into account the cases of death at early age, genotype-positive cases without phenotypic expression, and the cases with limited values of the LVH, where the diagnosis is controversial. Considering that information, the HCM frequency among the total population is probably about $0.5 \%$ (Semsarian et al., 2015).

It can be assumed that the frequent of HCM among the athletes is lower than that in the general population. This hypothesis is logical for several reasons: usually athletes are in childhood and young age, in which the incidence of the disease is lower; the early phenotypic evidence of the disease leads to cessation of active sporting activity; the existence of significant complaints in patients with $\mathrm{HCM}$ is incompatible with practicing of competitive sports, especially some of the sport disciplines, where very high aerobic performance is necessary.

\section{Etiology of hypertrophic cardiomyopathy}

The most common cause of HCM's development are gene mutations, which are transmitted as an autosomal dominantly trait. They affect the genes encoding sarcomere proteins and are responsible for $40-60 \%$ of all cases (Van Driest et al., 2005; Morita et al., 2008; Lopes et al., 2013). In the majority of cases, in genetically determined HCM, mutations of the genes encoding the beta-myosin heavy chain (MYH7) and myosin-binding protein $\mathrm{C}$ (MYBPC3) (70-80\%) are involved. The gene mutations rarely affect troponin $\mathrm{I}$ and $\mathrm{T}$, tropomyosin alpha-1 chain and myosin light chain 3 . These gene mutations are responsible for 50-70\% of all manifested HCM clinical cases (GeisterferLowrance et al., 1990; Watkins et al., 1993). Patients with proven gene mutations are more likely to have a positive family history and a higher risk of SCD (Coats et al., 2013; Olivotto et al., 2008). On the other hand, not all cases of gene mutations are phenotypically presented. There is still no clarity about the SCD risk in these individuals. Other causes of HCM development (according to the ESC expert group) include metabolic disorders (AndersonFabry disease, Danon disease), mitochondrial cardiomyopathies, neuromuscular diseases, infiltrative diseases, endocrine diseases, etc. (Elliott et al., 2014). It should not be forgotten that in about $25-30 \%$ of cases, the cause of HCM development is not identified. Probably, the determining role in HCM genesis in athletes has the genetic mutations. There are two main reasons that justify this assumption: their leading role in the general population, and incompatibility with elite sport in some of the other mentioned above diseases leading to hypertrophy. 


\section{Pathophysiology of hypertrophic cardiomyopathy}

The hypertrophic cardiomyopathy causes a number of pathophysiological changes that determined most of the symptoms and prognosis of patients. One of the leading hemodynamic disorders is the presence of gradient in the left ventricular outflow tract (LVOT). This is a peak gradient that occurs due to obstruction, caused by a septum hypertrophy, papillary muscle hypertrophy, mitral valve displacement, etc. Moreover, the obstruction is dynamic and depends on the preload and afterload of the ventricle, heart rate, contractility, and other factors. About 1/3 of the patients have gradient above $30 \mathrm{mmHg}$ at rest, another $1 / 3$ have a gradient below 30 $\mathrm{mmHg}$ at rest and above $30 \mathrm{mmHg}$ after provocation, and the remaining 1/3 have a gradient below $30 \mathrm{mmHg}$ at rest as well as after provocation (nonobstructive form of HCM) (Maron et al., 2006). Patients with gradient above $50 \mathrm{mmHg}$ at rest or after provocation are usually symptomatic and are indicated for surgical or interventional treatment.

Diastolic dysfunction is another significant pathophysiological disorder. It is due to compromised ventricular relaxation and increased myocardial rigidity due to intracellular calcium retention, myocardial hypertrophy and probable ischemia (Wigle et al., 1995).

The emergence of myocardial ischemia is not related to morphological changes in subepicardial coronary arteries. The leading cause is the imbalance between oxygen supply to myocardium and disproportionately increased oxygen demand (Maron et al., 2009). Compression of the intramural coronary arteries of the hypertrophied myocardium is also affected (Maron et al., 1986). These pathophysiological changes can lead to severe ischemia, including a myocardial infarction. Approximately $25 \%$ of the patients experience abnormal blood pressure response at exercise, and systolic levels may not rise by more than $20 \mathrm{mmHg}$ or decrease in some cases ( Frenneaux et al., 1990; Sadoul et al., 1997). This reaction is probably due to a suboptimal increase in cardiac output caused by an obstruction and systemic vasodilatation, which are induced by physical loading.

The mitral regurgitation is a common disorder in patients with obstructive HCM. It is a secondary occurrence because of the valve apparatus deformation, and is often dynamic (Wigle et al., 1995). In some cases, mitral regurgitation is hemodynamically significant.

It can be assumed that systemic physical workloads in athletes have radically opposite effects on the major pathophysiological disorders. On one hand, the intense physical loads can provoke increased left ventricular outflow gradient or myocardial ischemia. On the other hand, regular volume loading of the left ventricle during physical exercise may contribute to increasing its volume and improving the ventricular relaxation. 


\section{Symptoms in patients with hypertrophic cardiomyopathy}

The disease is asymptomatic in most patients, and in the most cases they have a normal life expectancy. It does not limit their physical working capacity, and it is possible for these individuals to practice competitive sports. Top sporting achievements are also possible. In some patients, the symptoms appear later in the evolution of the disease, years after its diagnosis. In other patients, symptom occurrence is the cause of HCM detection. The most common manifestations of HCM are fatigue and dyspnoea, chest pain, palpitations, presyncope and syncope, and SCD.

The fatigue and shortness of breath are relatively common symptoms and they are a manifestation of left-sided heart failure. In most cases, they are associated with diastolic dysfunction in the presence of a normal or a reduced left ventricular size and preserved systolic function. Rarely, in advanced cases of the disease, the left ventricle systolic function can be impaired. The presence of a hemodynamically significant gradient in the left ventricular outflow tract, and mitral insufficiency and rhythm disturbances, especially atrial fibrillation, may affect the appearance and severity of the symptoms. In athletes, the manifestations of heart failure are incompatible with competitive sports, especially in heavier sports disciplines.

Chest pain is another common symptom in HCM patients. It can occur at rest or during physical effort, and may be provoked after eating and excess alcohol consumption (Feiner et al., 2013; Paz et al., 1996). In the most cases, it is a myocardial ischemia associated with microvascular dysfunction, increased systolic myocardial stress, and a high gradient in the left ventricular outflow tract (Elliott et al., 2014).

Syncope is another manifestation of HCM. It is a typical symptom, but a possible vasovagal syncope or other mechanism, not associated with the disease, should be considered. Typical syncope in HCM patients is associated with various mechanisms: hypovolaemia, complete AV block, sinoatrial node dysfunction (Barriales-Villa et al., 2010), slow ventricular tachycardia, a sharp rise in the left ventricular outflow gradient (McCully et al., 1996), vascular dysregulation (Counihan et al., 1991; Prasad et al., 2008), and supraventricular arrhythmias with rapid ventricular response (Olivotto et al., 2001). It can be expected that in athletes, syncope may be the first and a relatively common manifestation of HCM, as they are not related to limiting of a physical capacity.

The SCD is the most serious manifestation of HCM. It is mainly due to primary ventricular fibrillation, and rarely there are cases of AV block, primary asystole and electromechanical dissociation (Nicod et al., 1988; Maron et al., 2000). The annual mortality of HCM patients is about $1 \%-2 \%$ (Elliott et al., 2006). Assessing SCD risk in HCM patients is essential for effective clinical behavior and lifestyle recommendations. Risk stratification 
is based on criteria that are received from anamnesis, physical examination, electrocardiography (ECG), Holter ECG, ECG working sample, echocardiography (EchoCG). According to AHA's recommendations in 2011, the main criteria for risk assessment are: ventricular tachycardia attacks, a maximum LV wall thickness over $30 \mathrm{~mm}$, a family history of SCD, unexplained syncope, and abnormal response of blood pressure response during effort (Gersh et al., 2011). A more recent risk stratification model, based on a multicenter retrospective study, was published in 2014 (O'Mahony et al., 2014). In addition to the aforementioned criteria (excluding abnormal blood pressure reaction), the following were added: left atrium diameter; maximal gradient in the LVOT, and age. In this model, the predictive value of the SCD for a five-year period has been significantly improved. Based on a SCD risk for a five-year period, the patients are divided into three groups $-\mathrm{a}$ low risk (less than $4 \%$ mortality), a moderate risk (4-6\% mortality) and a high risk (over 6\% mortality) (Elliott et al., 2014).

The risk of SCD in athletes with HCM is likely to be low in most cases, as many of the risk assessment criteria are incompatible with the active competitive sports. It should be taken into account that the disease is diagnosed earlier in a lot of the athletes (especially in connection with mandatory prophylactic examinations) and they are recommended to cease active participation in competitive sport. This is confirmed by the data on the low general SCD risk in athletes, estimated at around $1-3 / 100000$ athletes per year (Maron et al., 2009; Van Camp et al., 1995; Corrado et al., 2003; Harmon et al., 2011; Maron et al., 2014). The results are objective, although most of these studies identify the HCM as the most common cause for SCD.

\section{Diagnosis and differential diagnosis of hypertrophic cardiomyopathy}

It is relatively easy to diagnose HCM, because the basic diagnostic criteria is the left ventricular wall hypertrophy, which can be detected by different imaging methods; EchoCG, Computed Tomography, and Magnetic Resonance Tomography. The hypertrophy may affect one or more segments in the absence of causes for a ventricle tension loading (arterial hypertension, aortic stenosis, etc.). The phenotypic disease manifestation also includes myocardial fibrosis, abnormalities of the mitral valve apparatus, impaired coronary microcirculation, and ECG abnormalities (Elliott et al., 2014). The diagnosis is certain for adults with a left ventricular wall thickness $\geq 15 \mathrm{~mm}$, uncertain at values of $13-14 \mathrm{~mm}$, and requires additional criteria such as family burden, non-cardiac symptoms, ECG abnormalities, laboratory tests, genetic analysis, etc. The main diagnostic criteria in children is the left ventricular wall thickness above 2 standard deviations from the mean average.

Differential diagnostic problems arise in patients with arterial hypertension, valvular diseases, isolated septal hypertrophy in adults, and 
physiological hypertrophy in athletes. In these cases, a number of additional diagnostic criteria are of major importance for the diagnosis. One of the major challenges is the differential diagnosis between the LVH in athletes and HCM. Incorrect HCM diagnosis or missing this diagnosis in athletes may lead to serious health, psychological and socio-economic consequences. The biggest differential-diagnosis difficulties occur when the left ventricular wall thickness is 13-16 mm, the so-called "gray area". It is formed for two reasons: 1. Part of the patients with HCM have a wall thickness of the left ventricle below $15 \mathrm{~mm}$ (probably 15-20\%); 2. Some athletes have a LVH over $12 \mathrm{~mm}$, rarely reaching $16 \mathrm{~mm}$. The left-ventricular hypertrophy in athletes always should be critically evaluated, taking into account a sports discipline, sports qualification, stage of preparation, age, gender, race, and other factors. The significance of these factors is mentioned in a number of studies that have detected a left-ventricular hypertrophy over $12 \mathrm{~mm}$ in a small part of the athletes (about $2 \%$ of the white race and about $15 \%$ of the black race) (Pelliccia et al., 1991; Basavarajaiah et al., 2008). Most often, hypertrophy occurs in cyclists, rowers, and ski runners.

The differential diagnosis for athletes who fall in the "gray area" is based on data from a number of studies - current complaints, family history, ECG, EchoCG, ECG stress tests (including gas analysis), magnetic resonance tomography, and genetic tests. All of these methods can help to diagnose, but some of the parameters obtained may have greater differential-diagnosis value. Several studies have been conducted in this direction. Some of them compared the results of studies in athletes with a LVH to the HCM patients (Sharma et al., 2002; Vinereanu et al., 2001; Caselli et al., 2014; Grazioli et al., 2016). Other studies compare athletes with a LVH to sport active people with HCM, which is likely to lead to more correct conclusions (Sheikh et al., 2013; Malhotra et al., 2014). According to these studies, the most important differential-diagnosis criteria are the following: a relative septum thickness versus a left ventricular cavity diameter, inversion of the T-wave, spatial QRS$\mathrm{T}$ angle over 45 degrees, left ventricular cavity diameter, left atrium diameter, ratio of E-wave to A-wave of the mitral diastolic blood flow, and family burden. In the various studies, the severity of these parameters is assessed differently. The relative septum thickness and the left ventricular cavity diameter most likely have the greatest differential-diagnostic value.

\section{Conclusion}

One of the most significant issues concerns behaviors in athletes diagnosed with HCM. The correct decision relates to the following findings from studies that have already been completed: 1 . HCM is the most common or one of the most common causes of SCD in athletes; 2 . It is likely that the incidence of SCD in athletes with HCM is lower than in individuals with this 
disease who do not exercise; 3 . There is no reliable evidence that the SCD in athletes occurs more often during physical activity; 4. The risk stratification from a SCD shows that it is different for the individual HCM patients; 5. It is likely that physical exertions, on one hand, may increase the risk of the SCD, but on the other hand, they bring certain benefits in terms of the quality of life, and perhaps in terms of prognosis (Klempfner et al., 2015); 6. In genotypepositive persons, without phenotypic expression, the risk of SCD is very low; 7. Implantable cardioverter defibrillator is effective in prevention the SCD (Lampert et al., 2013). Taking into account all this information, it seems that the most appropriate behavior is the individual approach to every athlete. However, according to the recommendations of the European Cardiology Society, all athletes with HCM diagnosis, including phenotype-negative, should cease participating in active competitive sports (Elliott et al., 2014). The recommendations of the American Cardiology Association are more liberal (Maron et al., 2015): 1. Participation in competitive sports of HCM genotype-positive individuals without phenotypic expression is acceptable; 2 . Individuals with clinically proven HCM should not participate in competitive sports, except in those with low intensity. Perhaps in the future, on the basis of more precise risk stratification, an individual approach will be adopted for the HCM patients in terms of the sports activity.

\section{References:}

1. Barriales-Villa, R., Centurion-Inda, R., Fernandez-Fernandez, X., Ortiz, M., Perez-Alvarez, L., Rodriguez, G., Hermida-Prieto, M., Monserrat, L. (2010), Severe cardiac conduction disturbances and pacemaker implantation in patients with hypertrophic cardiomyopathy, Rev Esp Cardiol, Vol. 63, pp. 985-988.

2. Basavarajaiah, S., Boraita, A., Whyte, G. et al. (2008), Ethnic differences in left ventricular remodeling in highly-trained athletes relevance to differentiating physiologic left ventricular hypertrophy from hypertrophic cardiomyopathy, J Am Coll Cardiol, Vol.51 No.23, pp. 2256-62.

3. Caselli, S., Maron, M., Urbano-Moral, J., Pandian, N., Maron, B., Pelliccia, D. (2014), Differentiating left ventricular hypertrophy in athletes from that in patients with hypertrophic cardiomyopathy. Am J Cardiol, Vol.114, pp.1383-1389

4. Coats, C.J. and Elliott, P.M. (2013), Genetic biomarkers in hypertrophic cardiomyopathy. Biomark Med, Vol. 7, pp. 505-516.

5. Corrado, D., Basso, C., Rizzoli, G., et al. (2003), Does sports activity enhance the risk of sudden death in adolescents and young adults?, $J$ Am Coll Cardiol, Vol.42, pp. 1959-63. 
6. Counihan, P.J., Frenneaux, M.P., Webb, D.J., McKenna, W.J. (1991), Abnormal vascular responses to supine exercise in hypertrophic cardiomyopathy, Circulation, Vol. 84, pp. 686-696.

7. Elliott P.M., Anastasakis A., Borger M.A. et al. (2014), ESC Guidelines on diagnosis and management of hypertrophic cardiomyopathy. European Heart Journal, Vol. 35, pp. 2733-2779.

8. Elliott, P.M., Gimeno, J.R., Thaman, R., Shah, J., Ward, D., Dickie, S., Tome Esteban, M.T., McKenna, W.J. (2006), Historical trends in reported survival rates in patients with hypertrophic cardiomyopathy, Heart, Vol. 92, pp. 785-791.

9. Feiner, E., Arabadjian, M., Winson, G., Kim, B., Chaudhry, F., Sherrid, M.V. (2013), Post-prandial upright exercise echocardiography in hypertrophic cardiomyopathy, J Am Coll Cardiol, Vol. 61. pp. 2487-2488.

10. Frenneaux, M.P., Counihan, P.J., Caforio, A.L., et al. (1990), Abnormal blood pressure response during exercise in hypertrophic cardiomyopathy, Circulation, Vol. 82, pp. 1995-2002.

11. Geisterfer-Lowrance, A.A., Kass, S., Tanigawa, G. et al. (1990), A molecular basis for familial hypertrophic cardiomyopathy: a beta cardiac myosin heavy chain gene missense mutation, Cell, Vol. 62, pp. 999-1006.

12. Gersh, B.J., Maron, B.J., Bonow, R.O. et al. (2011), ACCF/AHA Guideline for the diagnosis and treatment of hypertrophic cardiomyopathy: Executive summary, Circulation, Vol. 124, pp. 2761-2796.

13. Grazioli, G., Usín, D., Trucco, E., et al. (2016), Differentiating hypertrophic cardiomyopathy from athlete's heart: An electrocardiographic and echocardiographic approach, $J$ Electrocardiol, Vol. 49 No. 4, pp. 539-44.

14. Harmon, K.G., Asif, I.M., Klossner, D., et al. (2011), Incidence of sudden cardiac death in national collegiate athletic association athletes, Circulation, Vol. 123, pp. 1594-600.

15. Klempfner, R., Kamerman, T., Schwammenthal, E., Nahshon, A., Hay, I., Goldenberg, I., Dov, F., Arad, M. (2015), Efficacy of exercise training in symptomatic patients with hypertrophic cardiomyopathy: results of a structured exercise training program in a cardiac rehabilitation center, Eur J Prev Cardiol. Vol. 22 No. 1, pp.13-9.

16. Lampert, R., Olshansky, B., Heidbuchel, H., et al. (2013), Safety of sports for athletes with implantable cardioverter-defibrillators, Circulation, Vol. 127, pp. 2021-2030.

17. Lopes, L.R., Zekavati, A., Syrris P., Hubank, M., Giambartolomei, C., Dalageorgou, C., Jenkins S., McKenna, W., Plagnol, V., Elliott P.M. 
(2013), Genetic complexity in hypertrophic cardiomyopathy revealed by high-throughputsequencing, J Med Genet Vol. 50, pp. 228-239.

18. Malhotra, A., Sheikh, N., Dhutia, H., et al. (2014), Differentiating physiological left ventricular hypertrophy from hypertrophic cardiomyopathy in athletes: proposed echocardiographic protocol, Heart, Vol. 100 (Suppl. 3): A52.

19. Maron, B., Udelson, J., Bonow, R., et al. (2015), Eligibility and Disqualification Recommendations for Competitive Athletes With Cardiovascular Abnormalities: Task Force 3: Hypertrophic Cardiomyopathy, Arrhythmogenic Right Ventricular Cardiomyopathy and Other Cardiomyopathies, and Myocarditis, Circulation, Vol. 132, pp. e273-e280.

20. Maron, B.J., Haas, T.S., Murphy, C.J., et al. (2014), Incidence and causes of sudden death in U.S. College athletes, $J$ Am Coll Cardiol, Vol. 63, pp.1636-43.

21. Maron, M.S., Olivotto, I., Maron, B.J., et al. (2009), The case for myocardial ischemia in hypertrophic cardiomyopathy, J Am Coll Cardiol, Vol. 54, pp.866-75.

22. Maron, B.J., Doerer, J.J., Haas, T.S., et al. (2009), Sudden deaths in young competitive athletes. Analysis of 1866 deaths in the United States, 1980-2006, Circulation, Vol. 119, pp.1085-92.

23. Maron, M.S., Olivotto, I., Zenovich, A.G., et al. (2006), Hypertrophic cardiomyopathy is predominantly a disease of left ventricular outflow tract obstruction, Circulation, Vol. 114, pp. 2232-9.

24. Maron, B.J. (2004), Hypertrophic cardiomyopathy: an important global disease, Am J Med, Vol. 116, pp. 63-5.

25. Maron, B.J., Olivotto, I., Spirito, P., Casey, S.A., Bellone, P., Gohman, T.E., Graham, K.J., Burton, D.A., Cecchi, F. (2000), Epidemiology of hypertrophic cardiomyopathy-related death: revisited in a large nonreferral-based patient population, Circulation, Vol. 102, pp. 858-864.

26. Maron, B.J., Wolfson, J.K., Epstein, S.E., et al. (1986), Intramural ("small vessel") coronary artery disease in hypertrophic cardiomyopathy, J Am Coll Cardiol, Vol. 8, pp. 545-57.

27. McCully, R.B., Nishimura, R.A., Tajik, A.J., Schaff, H.V., Danielson, G.K. (1996), Extent of clinical improvement after surgical treatment of hypertrophic obstructive cardiomyopathy, Circulation, Vol. 94, pp. 467-471.

28. Morita, H., Rehm, H.L., Menesses, A., McDonough, B., Roberts, A.E., Kucherlapati, R., Towbin, J.A., Seidman, J.G., Seidman, C.E. (2008), Shared genetic causes of cardiac hypertrophy in children and adults, $N$ Engl J Med, Vol. 358, pp.1899-1908. 
29. Nicod, P., Polikar, R., Peterson, K.L. (1988), Hypertrophic cardiomyopathy and sudden death, $N$ Engl J Med, Vol. 318, pp.12551257.

30. Olivotto, I., Girolami, F., Ackerman, M.J., Nistri, S., Bos, J.M., Zachara, E., Ommen, S.R., Theis, J.L., Vaubel, R.A., Re, F., Armentano, C., Poggesi, C., Torricelli, F., Cecchi, F. (2008), Myofilament protein gene mutation screening and outcome of patients with hypertrophic cardiomyopathy, Mayo Clin Proc, Vol. 83, pp. 630638.

31. Olivotto, I., Cecchi, F., Casey, S.A., Dolara, A., Traverse, J.H., Maron, B.J. (2001), Impact of atrial fibrillation on the clinical course of hypertrophic cardiomyopathy, Circulation, Vol. 104, pp. 2517-2524.

32. O’Mahony, C., Jichi, F., Pavlou, M., Monserrat, L., Anastasakis, A., Rapezzi, C., Biagini, E., Gimeno, J.R., Limongelli, G., McKenna, W.J., Omar, R.Z., Elliott, P.M. (2014), A novel clinical risk prediction model for sudden cardiac death in hypertrophic cardiomyopathy (HCM Risk-SCD), Eur Heart J, Vol. 35, pp. 2010-2020.

33. Paz, R., Jortner, R., Tunick, P.A., Sclarovsky, S., Eilat, B., Perez, J.L., Kronzon, I. (1996), The effect of the ingestion of ethanol onobstruction of the left ventricular outflow tract in hypertrophic cardiomyopathy, $N$ Engl J Med, Vol. 335, pp. 938-941.

34. Pelliccia, A., Maron, B.J., Spataro, A., et al. (1991), The upper limit of physiologic cardiac hypertrophy in highly trained elite athletes, $N$ Engl J Med, Vol. 324 No. 5, pp. 295-301.

35. Prasad, K., Williams, L., Campbell, R., Elliott, P.M., McKenna, W.J., Frenneaux, M. (2008), Episodic syncope in hypertrophic cardiomyopathy: evidence for inappropriate vasodilation, Heart, Vol. 94, pp.1312-1317.

36. Sadoul, N., Prasad, K., Elliott, P.M., et al. (1997), Prospective prognostic assessment of blood pressure response during exercise in patients with hypertrophic cardiomyopathy, Circulation, Vol. 96, pp. 2987-91.

37. Semsarian, C., Ingles, J., Maron, M.S., Maron, B.J. (2015), New perspectives on the prevalence of hypertrophic cardiomyopathy, $J$ Am Coll Cardiol, Vol. 65, pp. 1249-54.

38. Sharma, S., Maron, B.J., Whyte, G., et al. (2002), Physiologic limits of left ventricular hypertrophy in elite junior athletes: relevance to differential diagnosis of athlete's heart and hypertrophic cardiomyopathy, J Am Coll Cardiol, Vol.40 No. 8, pp. 1431-6.

39. Sheikh, N., Papadakis, M., Ghani, S., et al. (2013), Comparison of electrocardiographic criteria for the detection of cardiac abnormalities 
in elite black and white athletes, Circulation, Vol. 129 No. 16, pp. 1637-49.

40. Teare, D. (1958), Asymmetrical hypertrophy of the heart in young adults, Br Heart J, Vol. 20, pp. 1-8.

41. Van Camp, S.P., Bloor, C.M., Mueller, F.O., et al. (1995), Nontraumatic sports death in high school and college athletes, Med Sci Sports Exerc, Vol. 27, pp. 641-7.

42. Van Driest, S.L., Ommen, S.R., Tajik, A.J., Gersh, B.J., Ackerman, M.J. (2005), Yield of genetic testing in hypertrophic cardiomyopathy, Mayo Clin Proc, Vol. 80, pp.739-744.

43. Vinereanu, D., Florescu, N., Sculthorpe, N., et al. (2001), Differentiation between pathologic and physiologic left ventricular hypertrophy by tissue doppler assessment of long-axis function in patients with hypertrophic cardiomyopathy or systemic hypertension and in athletes, Am J Cardiol, Vol. 88 No. 1, pp. 53-8.

44. Watkins, H., MacRae, C., Thierfelder, L., et al. (1993), A disease locus for familial hypertrophic cardiomyopathy maps to chromosome 1q3, Nat Gen, Vol. 3, pp. 333-7.

45. Wigle, E.D., Rakowski, H., Kimball, B.P., et al. (1995), Hypertrophic cardiomyopathy: clinical spectrum and treatment, Circulation, Vol. 92, pp. $1680-92$. 